\title{
Mate choice evolution, dominance effects, and the maintenance of genetic variation
}

\author{
Laurent Lehmann ${ }^{\mathrm{a}, *}$, Lukas F. Keller ${ }^{\mathrm{b}}$, Hanna Kokko ${ }^{\mathrm{a}}$ \\ ${ }^{a}$ Laboratory of Ecological and Evolutionary Dynamic, Department of Biological and Environmental Sciences, P.O. Box 65, 00014, \\ University of Helsinki, Finland \\ ${ }^{\mathrm{b}}$ Zoology Museum, University of Zürich, CH-8057 Zürich, Switzerland
}

Received 11 May 2006; received in revised form 12 July 2006; accepted 25 July 2006

Available online 12 August 2006

\begin{abstract}
Female mate choice influences the maintenance of genetic variation by altering the mating success of males with different genotypes. The evolution of preferences themselves, on the other hand, depends on genetic variation present in the population. Few models have tracked this feedback between a choice gene and its effects on genetic variation, in particular when genes that determine offspring viability and attractiveness have dominance effects. Here we build a population genetic model that allows comparing the evolution of various choice rules in a single framework. We first consider preferences for good genes and show that focused preferences for homozygotes evolve more easily than broad preferences, which allow heterozygous males high mating success too. This occurs despite better maintenance of genetic diversity in the latter scenario, and we discuss why empirical findings of superior mating success of heterozygous males consequently do not immediately lead to a better understanding of the lek paradox. Our results thus suggest that the mechanisms that help maintain genetic diversity also have a flipside of making female choice an inaccurate means of producing the desired kind of offspring. We then consider preferences for heterozygosity per se, and show that these evolve only under very special conditions. Choice for compatible genotypes can evolve but its selective advantage diminishes quickly due to frequency-dependent selection. Finally, we show that our model reproduces earlier results on selfing, when the female choice strategy produces assortative mating. Overall, our model indicates that various forms of heterozygote-favouring (or variable) female choice pose a problem for the theory of sexual ornamentation based on indirect benefits, rather than a solution.
\end{abstract}

(C) 2006 Elsevier Ltd. All rights reserved.

Keywords: Female choice; Heterozygosity; Inbreeding depression; Dominance; Genetic variation; Sexual selection

\section{Introduction}

A much debated idea in the study of sexual selection is whether females gain indirect fitness benefits through mate choice (Kirkpatrick and Ryan, 1991; Kokko et al., 2006; Qvarnström et al., 2006). More ornamented males are expected to sire offspring of higher reproductive value, due to heritable mating success and/or enhanced viability of offspring (Møller and Alatalo, 1999; Jennions and Petrie,

\footnotetext{
${ }^{*}$ Corresponding author. Current address: Department of Genetics, University of Cambridge, UK. Tel.: + 1358919157702 ; fax: + 1358919157694 .

E-mail addresses: 11316@cam.ac.uk (L. Lehmann), hanna.kokko@helsinki.fi (H. Kokko).
}

2000; Eshel et al., 2002; Kokko et al., 2002), but this requires that some process maintains heritable variation in the traits in question. It is also increasingly recognized that female choice can be more multi-faceted than a simple quest to look for the best genotype. For example, female choice can be context-dependent (Qvarnström et al., 2000; Welch, 2003) so that the best mate for a given female may depend on the female's own genotype (Tregenza and Wedell, 2000; Zeh and Zeh, 2003; Mays and Hill, 2004; Neff and Pitcher, 2005).

This gives rise to a challenge: how should females choose, when there may be heritable variation in fitness (so that mating with attractive males who carry 'good genes' gives highly viable and attractive offspring) but there are simultaneously benefits of both dissimilarity and 
complementarity so that mating with males with 'compatible genes' may be advantageous (Colegrave et al., 2002; Hunt et al., 2004; Mays and Hill, 2004; Neff and Pitcher, 2005)? The genetic architecture of the genotypes that confer fitness benefits under the two scenarios is fundamentally different: choice for 'good genes' assumes additive gene action while choice for compatibility assumes overdominance or epistasis (i.e. non-additive genetic action). This difference could have important consequences: perhaps benefits from dominance through mate choice play a role in how genetic variation is maintained?

Mate preferences that produce a genetically diverse offspring generation could obviously increase the genetic diversity that is maintained at equilibrium (Neff and Pitcher, 2005), thus feeding back and reinforcing the evolution of choosiness itself. Neff and Pitcher (2005) suggest that this leads to a continuum between possible mating systems, where choice for compatible genes leads to an increase in genetic variation, and hence enhances the prospects for choice of males for the sake of good genes. A variation on this theme occurs when inbreeding has adverse effects on male condition (Saccheri et al., 2005) and consequently on his sexual attractiveness (Maynard Smith, 1956; Aspi, 2000; Höglund et al., 2002; Ahtiainen et al., 2004; Reid et al., 2003, 2005). If heterozygosity correlates not only with viability but also with sexual attractiveness, fixation of a single best genotype due to mate choice could be avoided, which in turn ensures continual variation in traits related to mate choice.

However, whether the mechanism just discussed can be maintained by selection is not trivial (Irwin and Taylor, 2000; Reinhold, 2002). To see why, consider a simplified genetic setting where condition is determined by only one locus, with two alleles $A$ and $a$. In a traditional good-genes scenario, $A A$ males are in best condition, followed by $A a$ and then $a a$. With a heterozygote advantage, $A a$ males perform best and are preferred by females. It is true that this preference creates a diversity of offspring $A A, A a$ and $a a$ thus maintaining genetic variation. However, this preference also suffers a cost of producing plenty of offspring of the wrong kinds ( $A A$ and $a a$ ). Thus, while heterozygote advantage means that there is probably more reason to choose, i.e. more genetic variation at equilibrium, choice itself becomes less accurate in terms of producing the desired types of offspring. Detailed tracking of the types of offspring produced is required to determine the net effect, and the answer obviously depends on the degree of genetic dominance present.

Here, our aim is to develop a comprehensive model of mate choice when fitness depends on dominance effects of two homologous genes. We employ two approaches to do so: first, we establish the conditions for the invasion of a mutant choice allele and, second, we use a population genetic model to follow the changes in genetic diversity of the population as a result of the introduction of the mutant choice allele. Equilibrium gene frequencies are tracked to investigate the feedback between the evolution of mate choice and genetic diversity. Our population genetic derivations allow examination of a variety of female choice strategies. We concentrate on the following: preferences for good genes, with two different treatments of heterozygous males (see defining female choice strategies, below); preference for heterozygous males per se; preference for compatible males; and assortative mating. The value of our study is that it allows us to compare the success of all the above strategies (and, if required, additional ones) in a single comprehensive framework. In particular, we clarify whether benefits of mate choice through dominant gene effects can aid in explaining the maintenance of costly female choice, as various studies currently express differing views on the subject (Mitton et al., 1993; Brown, 1997; Irwin and Taylor, 2000; Reinhold, 2002). We ask how a fixed level of mutational input translates into female choice under different scenarios of genetic dominance, and differential attractiveness of homozygous vs. heterozygous males. We also discuss the relative merits of preferences for heterozygous males in general, compared to genotypespecific preferences for compatible males.

\section{Model}

\subsection{Describing the life cycle}

In any natural population, viability is influenced by a multitude of loci and alleles (Rowe and Houle, 1996; Tomkins et al., 2004). Nevertheless, to enable us to track the coevolution of genetic variation and female choice, we have chosen to simplify the situation such that only one locus with two alleles, say $A$ and $a$, determines the condition of both males and females (see Table 1 for a list of symbols). Condition in turn determines viability, and in the case of males it can also have an influence on their sexual appearance. We introduce female choice by considering a second locus with two possible alleles, $B$ involving choosy behaviour and $b$ implying random mating. For simplicity, we assume additive gene action at this locus and no gene interactions with the viability locus. Since under additive gene action a locus with diploid inheritance is functioning like a haploid locus we let the choice locus obey haploid inheritance, with half the offspring inheriting the allele from the mother, and half from the father. We also assume semelparous individuals living in a population of infinite size. The events of the lifecycle occur in the following order:

(1) Viability selection occurs among juveniles. Irrespective of the sex, the viabilities of individuals are the same. That is, the viability of homozygotes for allele $A$ is $w_{A A}=1$, the viability of homozygotes $a a$ is $w_{a a}=1-s$ and the viability of heterozygote individuals is $w_{A a}=1-h s$, where $h$ is the coefficient of dominance. Choosing appropriate values of $h$ allows us to cover three different scenarios: (a) overdominance: heterozygous individuals are more viable than either type of homozygote $(h<0)$, (b) dominance: the viability of heterozygotes is 
Table 1

List of symbols used in the model

\begin{tabular}{|c|c|}
\hline Symbols & Definitions \\
\hline$A, a$ & Alleles at the condition-determining locus \\
\hline$B, b$ & $\begin{array}{l}\text { Alleles at the choice locus, } B \text { determines } \\
\text { choosiness }\end{array}$ \\
\hline$p$ & Frequency of allele $A$ \\
\hline$q$ & Frequency of allele $B$ \\
\hline$\hat{p}$ & Equilibrium frequency of allele $A$ \\
\hline$p_{j}$ & Frequency of genotypes $j$ \\
\hline$w_{j}$ & Viability of genotypes $j$ \\
\hline$s$ & Coefficient of selection against $a$ \\
\hline$h$ & Coefficient of dominance \\
\hline$\mu$ & Mutation rate from allele $A$ to allele $a$ \\
\hline$v$ & Mutation rate from allele $a$ to allele $A$ \\
\hline$c$ & Cost of choice \\
\hline$\psi(k \mid j)$ & $\begin{array}{l}\text { Probability that a female with genotype } j \text { accepts } \\
\text { a randomly encountered male with genotype } k \\
\text { for mating }\end{array}$ \\
\hline$\psi$ & $\begin{array}{l}\text { Strategy profile: set of the probabilities } \psi(k \mid j) \\
\text { determined by allele } B\end{array}$ \\
\hline $\log \lambda$ & Choice gene growth rate when rare \\
\hline$\lambda$ & Dominant eigenvalue of the transition matrix $\mathbf{T}_{\hat{p}}$ \\
\hline $\mathbf{p}_{\mathrm{A}}$ & $\begin{array}{l}\text { Vector of genotype frequencies of the condition- } \\
\text { determining locus }\end{array}$ \\
\hline $\mathbf{p}_{\mathbf{B}}$ & $\begin{array}{l}\text { Vector of genotype frequencies of the condition- } \\
\text { determining locus } \\
\text { and choice allele } B\end{array}$ \\
\hline $\mathbf{p}$ & Vector of all genotype frequencies \\
\hline $\mathbf{T}$ & Transition matrix of genotype frequencies \\
\hline $\mathbf{W}$ & Viability matrix \\
\hline $\mathbf{M}$ & Mating matrix under choice \\
\hline $\mathbf{R}$ & Random mating matrix \\
\hline $\mathbf{U}$ & Mutation matrix \\
\hline$L \equiv 1-\frac{\bar{w}}{w_{\max }}$ & Genetic load \\
\hline$F_{I S} \equiv 1-\frac{p_{A a}}{2 p(1-p)}$ & Heterozygote deficiency index \\
\hline
\end{tabular}

somewhere in between the high-quality homozygotes and the low-quality homozygotes $(0<h<1)$, and (c) underdominance: heterozygous individuals are less viable than both types of homozygotes $(h>1)$. This formulation, however, cannot handle the particular case of symmetric overdominance, where either type of homozygote is equally strongly selected against. In some of our results, therefore, we use an alternative formulation for the explicit case of symmetric overdominance. In that case, the viability of heterozygotes is $w_{A a}=1$, while either type of homozygote has reduced fitness, $w_{A A}=1-s$ and $w_{a a}=1-s$.

(2) Juveniles become adults, and mating occurs. Males and females encounter each other in random order, and randomly mating females (allele $b$ ) accept the first male encountered. Choosy females (allele $B$ ) either accept or reject males for mating, depending on the genetically controlled preference. The probability that a female with genotype $j$ accepts a randomly encountered male with genotype $k$ for mating is denoted by $\psi(k \mid j)$. Thus, the model allows us to consider e.g. cases where $A A$ and $A a$ males have identically elaborate sexual displays, while $a a$ males perform much worse; in that case, females are expected to have equally high acceptance probabilities for $A A$ and $A a$, but low probabilities for $a a$ males. If a female rejects a male, she searches for a new mate until she has mated. There are no limits to how many females a male can inseminate, and all females are assumed to find an acceptable mate eventually. We do not consider maladaptive strategies where all acceptance probabilities are zero.

(3) Females produce a large number of juveniles with a 1:1 primary sex-ratio. The fertility of a choosy female is reduced by a factor $c$ relative to that of random mating females. For simplicity we thus assume a constant cost of expressing mate preferences, regardless of the actual number of males sampled. All adults die after breeding.

(4) Mutations occur. We assume that allele $A$ mutates to allele $a$ with probability $\mu$ while the back mutation rate from $a$ to $A$ is given by $v$.

\subsection{Dynamic of the viability genes}

We will first consider the dynamic of the conditiondetermining genotypes without the dynamic of female choice. This assumption will be relaxed later. Since we consider only a 1:1 primary sex ratio and segregation of viability alleles is assumed to occur at an autosomal locus, it is sufficient to consider only the dynamic of female genotypes. The frequency of genotype $j$ will be designated by $p_{j}$. Then, the frequencies of all female genotypes in the population at the juvenile stage can be collected into the column vector $\mathbf{p}_{\mathbf{A}} \equiv\left(p_{A A}, p_{A a}, p_{a a}\right)$. The frequency of genotypes in the next generation $\mathbf{p}_{\mathbf{A}^{\prime}}$ can be calculated from the frequencies in the previous generation according to the recursion

$\mathbf{p}_{\mathbf{A}^{\prime}}=\mathbf{U M W} \mathbf{p}_{\mathbf{A}}$.

The viability matrix $\mathbf{W}$ is diagonal and has elements $w(j \mid j)$ giving the relative viability of a female of genotype $j$ during viability selection. The mating matrix $\mathbf{M}$ has elements $\operatorname{Pr}(i \mid j)$ giving the probability that a female of genotype $j$ produces an offspring of genotype $i$. Finally, the mutation matrix $\mathbf{U}$ has elements $u(l \mid i)$ giving the probability that an offspring inheriting genotype $i$ will actually be of genotype $l$ after mutation (see Appendix). The relative viability of genotype $j$ is

$w(j \mid j)=\frac{w_{j}}{\sum_{r} p_{r} w_{r}}$,

where $w_{j}$ is the viability of genotype $j$. Accordingly, the frequency of genotype $j$ after viability selection is given by

$p_{j}^{\mathrm{s}}=\frac{p_{j} w_{j}}{\sum_{r} p_{r} w_{r}}$

and this equation holds for both male and female genotypes. The probability that a female of genotype $j$ produces an offspring of genotype $i$ can be expanded in terms of the various genotypes of her mating partners,

$\operatorname{Pr}(i \mid j)=\sum_{k} \operatorname{Pr}(i \mid k, j) \operatorname{Pr}(k \mid j)$, 
where $k$ runs over all male genotypes. The first term in this sum, $\operatorname{Pr}(i \mid k, j)$, is the probability that a female with genotype $j$ that has mated with a male with genotype $k$ produces an offspring of genotype $i$. This probability is obtained by applying the rules of Mendelian inheritance. The second term in the sum is the probability that a female with genotype $j$ mates with a male with genotype $k$ after viability selection and is given by

$\operatorname{Pr}(k \mid j)=\frac{p_{k}^{\mathrm{s}} \psi(k \mid j)}{\sum_{r} p_{r}^{\mathrm{s}} \psi(r \mid j)}$.

For completeness, we mention that the relative mating success of a male of genotype $k$ is $\left[\sum_{j} p_{j}^{\mathrm{s}} \operatorname{Pr}(k \mid j)\right] / p_{k}^{\mathrm{s}}$.

\subsection{Random mating}

An important factor to consider before introducing the choice gene locus is the genetic diversity available under random mating, i.e. the initial conditions which the choice allele experiences when attempting invasion. These initial conditions are determined by the equilibrium frequency $\hat{p}$ of allele $A$ under random mating. In this situation we have $\psi(k \mid j)=1$ for all male genotypes $k$ and female genotype $j$ and we designate the mating matrix under this specific assumption by $\mathbf{R}$. Using the Hardy-Weinberg proportions, the vector of the frequencies of female genotype at the juveniles stage is $\mathbf{p}_{\mathbf{A}}=\left(p^{2}, 2 p(1-p),(1-p)^{2}\right)$ where $p$ is the frequency of allele $A$ in the population. Using Eq. (1) and noting that $p=p_{A A}+p_{A a} / 2$, the change in the frequency of allele $A$ is

$\Delta p=\mathbf{p}_{\mathbf{A}^{\prime}} \cdot \mathbf{v}-p$,

where the vector $\mathbf{v} \equiv\left(1, \frac{1}{2}, 0\right)$ weights the contribution of each genotype to the frequency of allele $A$ (. is the dot product). Eq. (6) is in fact equivalent to the standard equation describing allele frequency change under random mating through the joint effect of selection and mutation (e.g. Hartl and Clark, 1997; Gillespie, 2004). Introducing the viabilities defined in the life-cycle into the random mating matrix, we can find the equilibrium frequency of allele $A$, which is reached when $\Delta p=0$. In the absence of backward mutations $(v=0)$ the stable equilibrium of allele $A$ is given by

$\hat{p}=\frac{2-h(3-\mu)-\sqrt{[4 \mu(1-2 h)] / s+h^{2}(1+\mu)^{2}}}{2(1-2 h)}$.

In the absence of mutations $(\mu=0)$, selection will drive the allele frequency towards $\hat{p}=1$ when $h \geqslant 0(h<0)$ or to $\hat{p}=$ $[1-h] /[1-2 h]$ in the presence overdominance. The equilibrium gene frequency (7) is plotted in Fig. 1A as a function of dominance $h$ for various values of the mutation rate $\mu$ and the coefficient of selection $s$. In order to relate the genetic architecture of the trait to the heritability maintained at steady state under random mating we also evaluated both the additive and dominance genetic variance in fitness at the viability locus as is usually carried

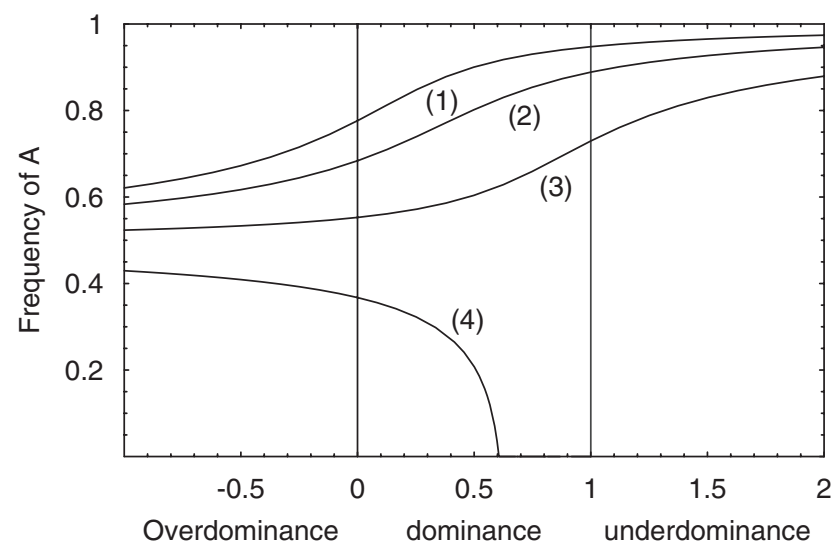

(A)

$\mathrm{h}$

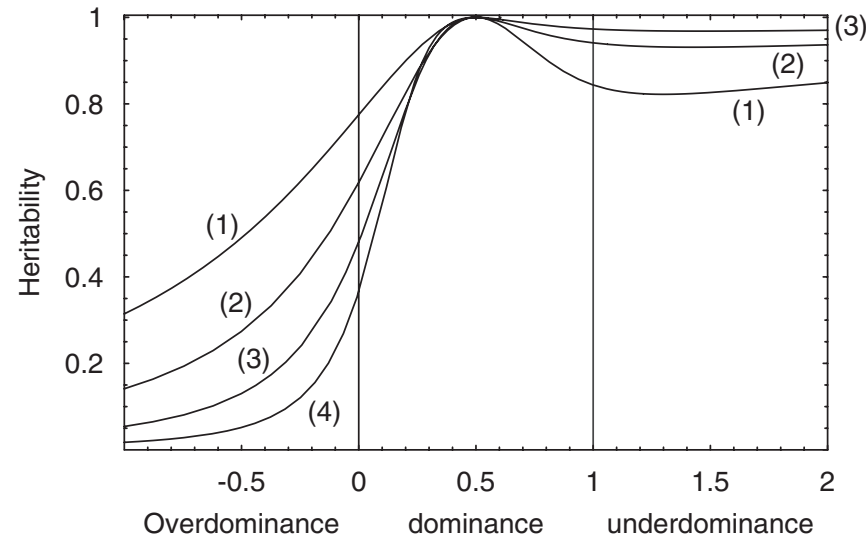

(B)

$\mathrm{h}$

Fig. 1. (A) Polymorphic equilibrium of the frequency $\hat{p}$ of allele $A$ under random mating (Eq. (7)) as a function of the dominance coefficient $h$. The four different curves correspond, from top to bottom, to: (1) $s=0.1$ and $\mu=0.005$, (2) $s=0.1$ and $\mu=0.01$, (3) $s=0.05$ and $\mu=0.01$ and (4) $s=0.025$ and $\mu=0.01$. A decrease in the coefficient of selection $s$ and an increase in the mutation rate $\mu$ decreases the equilibrium frequency of allele $A$. (B) Corresponding heritability at the viability locus (Eq. (8)). The four different curves correspond to the same parameter values as given in panel A.

out for quantitative traits (e.g. Bürger, 2000; Lynch and Walsh, 1998). The resulting variances in viabilities are $\sigma_{A}^{2}=2 \hat{p}(1-\hat{p}) s^{2}\{1-\hat{p}-h(1-2 \hat{p})\}^{2}$ and $\sigma_{D}^{2}=\hat{p}^{2}(1-\hat{p})^{2}$ $s^{2}(1-2 h)^{2}$ with the result that the heritability at the viability locus is given by

$$
\begin{aligned}
H^{2} & =\frac{\sigma_{A}^{2}}{\sigma_{A}^{2}+\sigma_{D}^{2}} \\
& =\frac{2(1-\hat{p}-h(1-2 \hat{p}))^{2}}{2-\hat{p}(3-\hat{p})-4 h(1-\hat{p})^{2}+h^{2}(2-4 \hat{p}(1-\hat{p}))},
\end{aligned}
$$

which is plotted in Fig. 1B as a function of dominance $h$ for various values of the mutation rate $\mu$ and the coefficient of selection $s$. The heritability takes a maximum value of one $\left(H^{2}=1\right)$ in the presence of additive gene action $\left(h=\frac{1}{2}\right)$ and thus decreases with dominance, overdominance and underdominance.

The stable equilibrium of allele $A$ can also be obtained for the case of symmetric overdominance (see stage 1 of the 
life cycle, above), when $v=0$ this is

$\hat{p}=\frac{1}{4}\left[3-\mu-\sqrt{[4 \mu(1-s)] / s+(1-\mu)^{2}}\right]$.

In the absence of mutations $(\mu=0)$, the equilibrium frequency of allele $A$ under symmetric overdominance is $\hat{p}=\frac{1}{2}$ for all selection coefficients $s$. The heritability at the viability locus for symmetric overdominance is given by

$H^{2}=\frac{1-4 \hat{p}(1-\hat{p})}{1-2 \hat{p}(1-\hat{p})}$.

Two other quantities will be important in our model. First, irrespective of her mating strategy, the probability that a heterozygote mother produces a heterozygote offspring is

$\operatorname{Pr}(A a \mid A a)=1 / 2$.

Second, the probability that a randomly sampled homozygote female from the population produces a heterozygote offspring is

$$
\begin{aligned}
\operatorname{Pr}(A a \mid A A \cup a a)= & \operatorname{Pr}(A a \mid A A) \frac{p_{A A}}{p_{A A}+p_{a a}} \\
& +\operatorname{Pr}(A a \mid a a) \frac{p_{a a}}{p_{A A}+p_{a a}},
\end{aligned}
$$

which is the proportion of heterozygote offspring produced by each homozygote mother averaged over the relative number of homozygotes in the population. Under random mating this is

$\operatorname{Pr}(A a \mid A A \cup a a)=\frac{(1-p) p}{1-2(1-p) p}$,

which corresponds to the equation in Mitton et al. (1993). This probability takes its maximum value of one half when the frequency of allele $A$ is one half $\left(p=\frac{1}{2}\right)$ in the population. Under the specific assumption that homozygote females mate only with heterozygote males, the probability that a randomly sampled homozygote female from the population produces a heterozygote offspring is

$\operatorname{Pr}(A a \mid A A \cup a a)=1 / 2$.

\subsection{Evolution of mate preferences}

Now that we have derived the equilibrium frequencies for condition-determining alleles of the viability locus, we must next determine if mate preferences can evolve. We assume that the mutant allele $B$ causes females to mate non-randomly according to a choice rule determined by the set $\boldsymbol{\psi} \equiv\{\psi(A A \mid A A), \ldots, \psi(k \mid j), \ldots, \psi(a a \mid a a)\}$ of acceptance probabilities. We will determine the invasion of allele $B$ in two stages. First, we evaluate whether the choice allele $B$, which imposes a fecundity cost, can invade a population fixed for allele $b$ when the equilibrium frequency of allele $A$ is held constant. Second, we track the coevolution of female choice and the condition-determining alleles during the spread of allele $B$. This allows us to answer questions relating to the maintenance of genetic diversity in the population. In the first stage of our analysis we follow previous work (Irwin and Taylor, 2000) and measure the ability of the mutant gene $B$ to invade the population by its growth rate relative to that of the established type in a population which has reached the mutation-selection balance at the condition-determining locus (e.g., equilibrium frequency $\hat{p}$ of allele $A$ at the viability locus given by Eq. (7) and heritability at this locus given by Eq. (8)). The frequency of choice gene carriers among juveniles of each genotype in the present generation is given by the column vector $\mathbf{p}_{\mathbf{B}} \equiv\left(p_{A A B}, p_{A a B}, p_{a a B}\right)$. Assuming that the genotype frequencies at the condition-determining locus do not change during the initial invasion of the mutant, the frequency of choice gene carriers in the next generation can be calculated from the frequency in the previous generation according to the recursion

$\mathbf{p}_{\mathbf{B}^{\prime}}=\mathbf{T}_{\hat{\mathbf{p}}} \mathbf{p}_{\mathbf{B}}$.

The subscript of the transition matrix $\mathbf{T}_{\hat{\mathbf{p}}}=(1-c) \mathbf{U} \mathbf{M}_{\hat{\mathbf{p}}} \mathbf{W}_{\hat{\mathbf{p}}}$ emphasizes that the elements of this matrix are evaluated at the random mating selection-mutation equilibrium $\hat{p}$ of allele $A$. The fate of an allele determining a choice rule $\psi$ is established by examining the dominant eigenvalue $\lambda$ of the transition matrix $\mathbf{T}_{\hat{\mathbf{p}}}$ (Caswell, 2001, p. 294). Indeed, the growth rate, defined as the logarithm of the dominant eigenvalue, of the random-mating allele $b$ is zero because the eigenvalue of the associated mating matrix is one. This is a direct consequence of using the equilibrium value $\hat{p}$ of the gene frequency which determines an evolutionary endpoint under random mating. Thus, when $\log \lambda>0$ under non-random mating, the choice allele is able to invade the population. By contrast, when $\log \lambda<0$, the choice allele will be wiped out of the population. The condition for the invasion of a mutant choice allele as given by the examination of the dominant eigenvalue greatly simplifies the analysis, but considering the initial prospects of invasion is not sufficient for all our questions. To track the feedback between choice and genetic diversity we proceeded to the second stage of the analysis, constructing a population genetic model. This allows us to check the validity of the invasion criteria and track the subsequent dynamic of the invasion. In this second stage of the analysis, we used a population genetic model to track the change of the genetic structure of the population as a result of the introduction of the mutant choice allele. With our system of inheritance we must track six genotypes in the population. The frequency of all genotypes at the juveniles stage is collected into the vector $\mathbf{p} \equiv\left(p_{A A B}, p_{A a B}, p_{a a B}, p_{A A b}\right.$, $\left.p_{A a b}, p_{a a b}\right)$ and we posit free recombination. The dynamic of genotypes satisfies the recursion

$\mathbf{p}^{\prime}=\mathbf{T} \mathbf{p}$,

where the transition matrix $\mathbf{T}$ describes the projection of the frequencies of genotypes from one generation to the next and is itself a function of genotype frequencies. This transition matrix is directly built on the elements presented so far, and its details are described in the appendix. 
As for the static model presented above, the mutant allele is introduced at low frequency into the population which is at equilibrium frequency for the conditiondetermining locus. The introduction is performed by changing the frequency of each of the three genotypes to a set of two frequencies, one for the choice allele $B$ carriers, the other for the random mating allele $b$ carriers. To avoid any initial association between choice gene and conditiondetermining genes, the frequencies of the mating type allele were initially assigned the same value within each of the genotype class at the condition-determining locus (Charlesworth et al., 1990). We subsequently report the dynamic of the frequency of allele $A$, the frequency of the choice allele $B$, and the frequency of heterozygotes. In addition, we followed the change in the genetic load of the population defined by $L \equiv 1-\bar{w} / w_{\max }$ where $w_{\max }$ is the viability of the best genotypes in the population and $\bar{w}$ is the mean viability (Gillespie, 2004). This index reflects the degree to which a choice allele can exploit the genetic variance in viability in the population to extract a fitness advantage over a random mating allele. When there is only one allele, $A$ or $a$, fixed in the population, $L=0$, and choice is not possible. Finally, we also followed the dynamic of the heterozygote deficiency index within a population as given by $F_{\text {IS }} \equiv 1-p_{A a} /[2 p(1-p)]$ where $2 p(1-p)$ is frequency of heterozygotes expected under random mating. This index reflects the degree to which the population is separated in different mating pools (Hartl and Clark, 1997; Gillespie, 2004; Gavrilets, 2004). Here, when $F_{\text {IS }}=1$ the population produces no heterozygotes and is therefore split into two reproductively isolated pools.

\subsection{Defining female choice strategies}

Although our model is general such that it allows us to consider all possible female preferences that satisfy the set form of acceptance probabilities, we derived results only for the following five biologically meaningful strategies:

(1) Focused preference for good genes. Here, we assume that females can distinguish $A A$ males from $A a$ or $a a$ males and mate only with $A A$ males. The preference profile is $\psi=\{\psi(A A \mid j)=1, \psi(A a \mid j)=0$ and $\psi(a a \mid j)=0$ for all $j\}$.

(2) Broad preference for good genes. Females can distinguish $a a$ males from the two other types and thus avoid breeding with them, but they cannot distinguish between $A A$ homozygotes and $A a$ heterozygotes. The acceptance probabilities are given by $\psi=\{\psi(A A \mid j)=1$, $\psi(A a \mid j)=1$ and $\psi(a a \mid j)=0$ for all $j\}$.

(3) Preference for heterozygotes. Regardless of her own genotype, each female chooses $A a$ males to mate with. The acceptance probabilities are given by $\psi=\{\psi(A A \mid j)=0$, $\psi(A a \mid j)=1$ and $\psi(a a \mid j)=0$ for all $j\}$.

(4) Disassortative mating. We assume here that a female knows both her own genotype and that of any potential mate. She only accepts $A A$ males if she is herself $a a$, accepts aa males if she is $A A$, and mates randomly if she is heterozygous, $A a$. The strategy profile is given by $\psi=$
$\{\psi(A A \mid A A)=0, \psi(A a \mid A A)=0, \psi(a a \mid A A)=1, \psi(a a \mid a a)$ $=0, \psi(A a \mid a a)=0, \psi(A A \mid a a)=1$ and $\psi(j \mid A a)=1$ for all $j\}$. Disassortative mating in our one-locus case can also be interpreted as achoice for compatible genotypes, if heterozygotes are more fit.

(5) Assortative mating. Again, we assume that females know their own genotype, and mate with males that have the same genotype as herself. The acceptance probabilities are given by $\psi=\{\psi(A A \mid A A)=1, \psi(A a \mid A a)=$ $1, \psi(a a \mid a a)=1$, else $\psi(l \mid j)=0\}$. Note that our model allows us to combine any pattern of dominance freely with any female choice strategy. Some combinations, of course, make more biological sense than others: for example, if there is overdominance in condition and sexual traits are condition-dependent, a female who pays attention to a male sexual trait is adequately described by strategy (3). This strategy would require much more complicated cognitive mechanisms, however, if condition was governed by intermediate dominance $(0<h<1)$, and under genetic underdominance, females would actually have to prefer poorly signalling males to achieve strategy (3). We do not, however, a priori exclude any combination. Instead, we investigated the success of each strategy for the whole continuum of the three different scenarios of overdominance, dominance and underdominance. It is worth keeping the biological feasibility in mind when interpreting model results, and also that grossly (or even mildly) maladaptive strategies will not spread or become fixed when their success is tracked in the model.

\section{Results}

\subsection{Preferences for good genes when fitness is non-additive}

Here, we examine the two strategies that aim to increase the chances that the offspring have the beneficial $A$ allele: (1) focused preferences for good genes, and (2) broad preferences for good genes. Assuming suitable values of selection and mutation from allele $A$ to allele $a$ (for simplicity we neglect mutations from $a$ to $A$ ) to ensure some genetic diversity at the random mating equilibrium, focused preferences can invade a randomly mating population when there is dominance or underdominance, but not if there is strong overdominance (Fig. 2). This result is easy to explain. If overdominance is strong, random mating performs better than a preference for $A A$ homozygotes, as the latter fails to produce fit heterozygous offspring. In the more favourable cases of dominance or underdominance, introducing costs of female choice counteract the indirect benefit of choice, but do not destroy it assuming that costs remain small (Fig. 2). This is the essence of the genic capture hypothesis, phrased in our simplified two-allele form: a sufficient mutational input allows costly female choice to invade and persist in the population (Fig. 3). The result that focused preferences can persist when $h>0$ is good news regarding the maintenance 

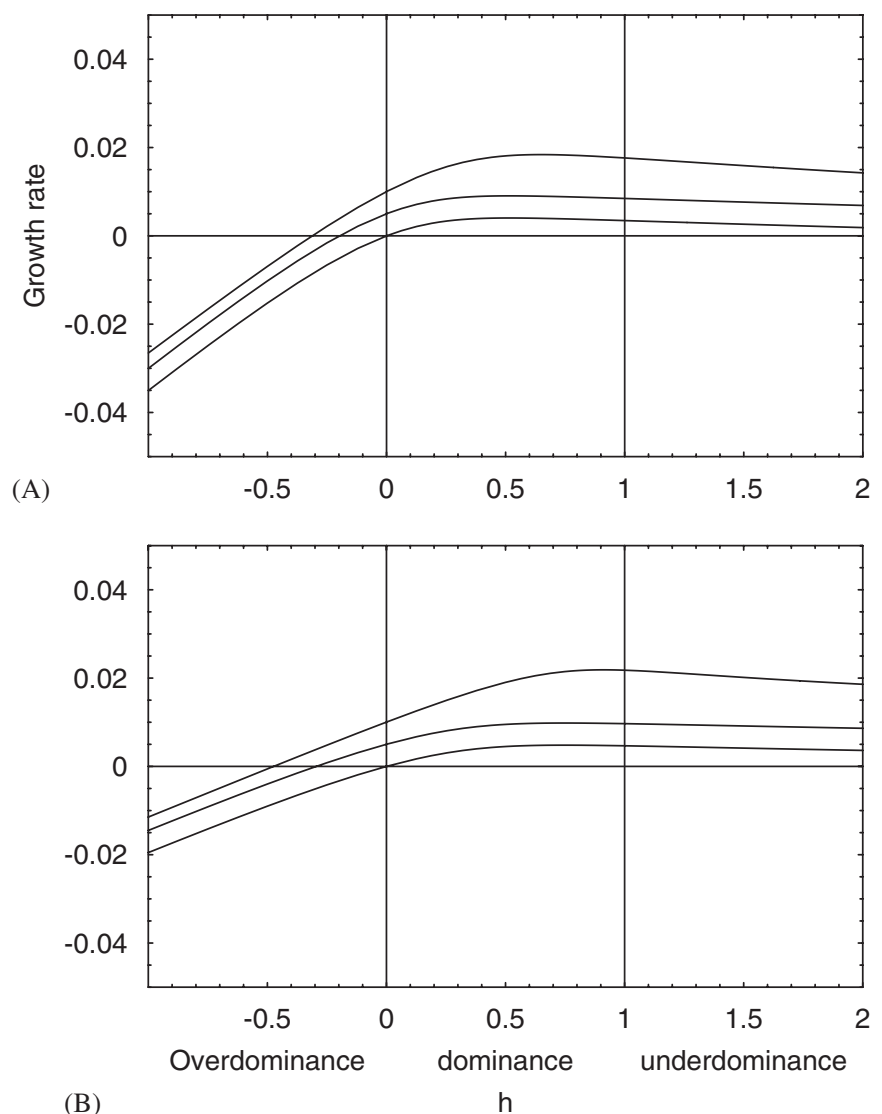

Fig. 2. Growth rate given by $\log \lambda$ of the strategy "focused choice for good genes" as a function of the dominance coefficient $h$. (A) The coefficient of selection is set to $s=0.1$ and the three curves correspond, from top to bottom, to: (1) $\mu=0.01$ and $c=0$, (2) $\mu=0.005$ and $c=0$, (3) $\mu=0.005$ and $c=0.005$. A decrease in the mutation rate and an increase in the cost of choice decreases the growth rate. (B) Same parameter values as (A) except the selection coefficient is set to $s=0.005$.

of female choice: preferences that focus on finding $A A$ males and make females avoid mating with heterozygotes are biologically more easily achieved by females when heterozygotes are phenotypically in worse condition than high-quality homozygotes (i.e., $h>0$ ).

It is interesting to compare "focused choice for good genes" with a broader form of preference, where females do not (or cannot) distinguish between $A A$ and $A a$ males. Does this situation help to maintain more diversity and more female choice? Note that biologically, the broad choice strategy (2) makes most sense when $A A$ and $A a$ males resemble each other in their condition, i.e. when $h$ is close to 0 . The prospects for choice to spread are, however, relatively insensitive to the exact value of $h$ (Fig. 3). When female choice is cost-free, broad choice can invade in all dominance scenarios: underdominance, dominance and overdominance (Fig. 3). This contrasts with the more restrictive setting of focused female choice, and thus, at first sight, overdominance seems to help contributing to the maintenance of female choice. However, the benefit is very slight (compare the $y$-axis in Fig. 3 to those in Fig. 2) and broad, diversity-maintaining choice rules are more sensitive
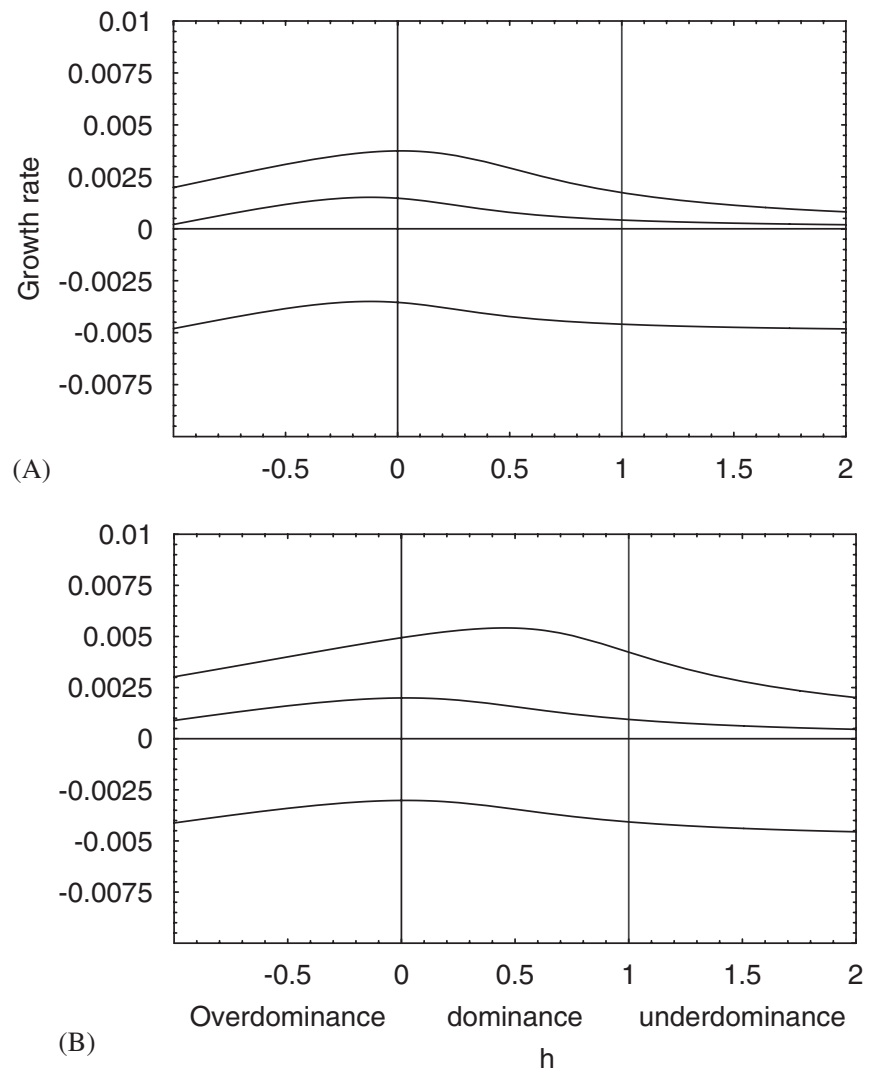

Fig. 3. Growth rate of the choice allele $B$ given by $\log \lambda$ of the strategy "broad choice for good genes" as a function of the dominance coefficient h. The parameters are the same as in fig. (2). (A) The coefficient of selection is set to $s=0.1$ and the three curves correspond, from top to bottom, to: (1) $\mu=0.01$ and $c=0$, (2) $\mu=0.005$ and $c=0$, (3) $\mu=0.005$ and $c=0.005$. A decrease in the mutation rate and an increase in the cost of choice decreases the growth rate. (B) Same parameter values as (A) except the selection coefficient is set to $s=0.005$.

to costs of female choice and thus evolve poorly under any scenario of dominance (Fig. 3, lowest curves).

Fig. 4 shows that the broad choice strategy indeed has the proposed advantage of maintaining more variation at the viability loci at equilibrium. In Figs. 4A-B, female choice is cost-free, and either focused (Fig. 4A) or broad (Fig. 4B) female choice can spread. Diversity is retained to a far greater degree in the latter scenario: as the choice allele $B$ spreads, the $A$ allele becomes close to fixation when female choice is focused, but not when it is broad. This is expected because focused choice is a much better strategy at picking out the favourable allele and transmitting it to offspring. Adding a slight cost to female choice does not destroy selection for the spread of the choice allele $B$ when it only favours $A A$ males (Fig. 4C), but $B$ consistently declines in frequency when choice is broad (Fig. 4D) despite the high genetic diversity present in the population.

\subsection{Preferences for heterozygotes vs. disassortative mating}

As exemplified in Fig. 5A, a preference for heterozygous males is unable to invade a population of randomly mating 

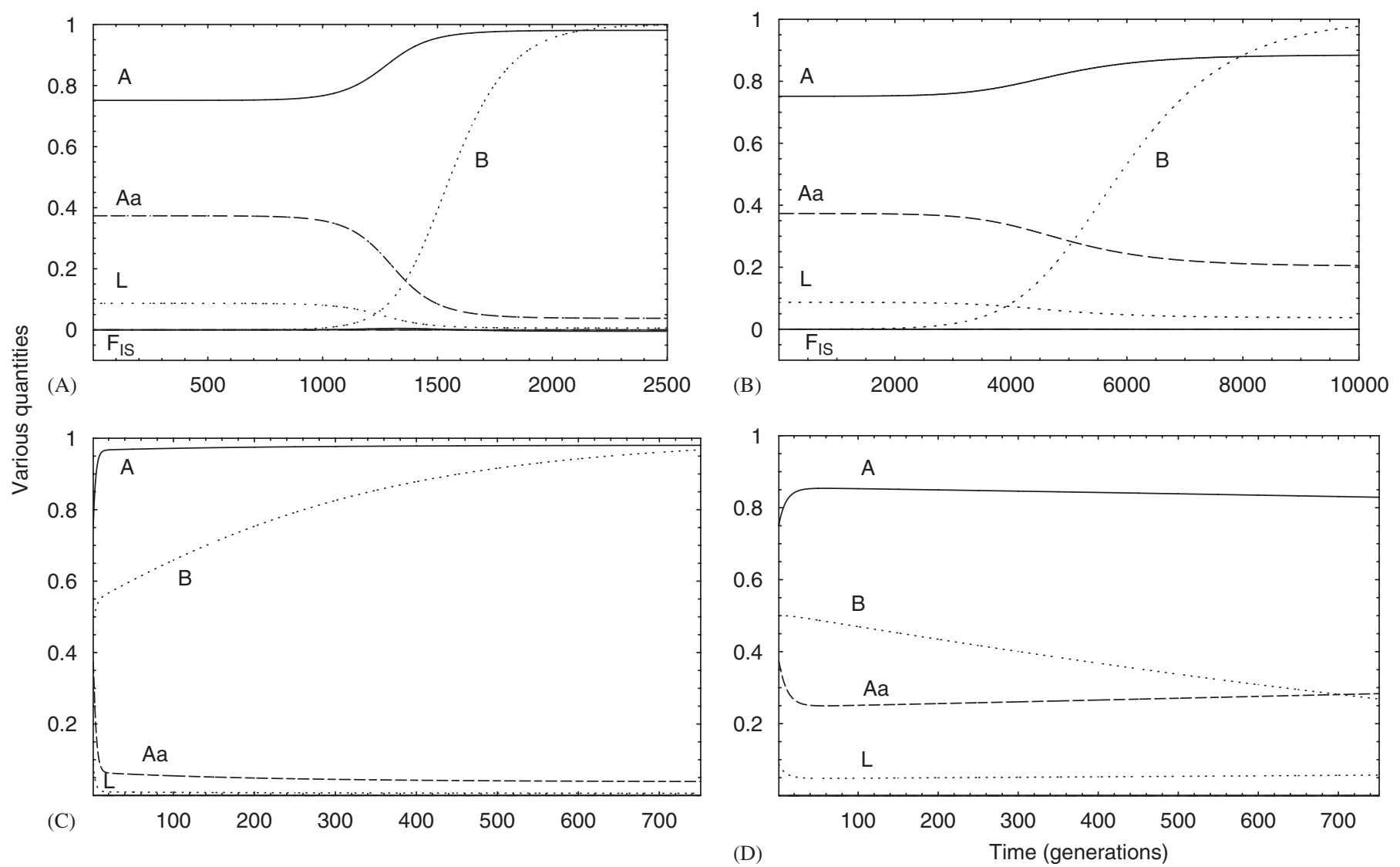

Fig. 4. Dynamic of allele frequencies, genetic load and heterozygote deficiency index as a function of time since the introduction of allele $B$ at frequency $q(0)$ in a population at equilibrium frequency $\hat{p}$ of allele $A$ (Eq. (7)). The plain line correspond to the frequency of allele $A$, the dashed line is the frequency of heterozygotes $A a$, the decelerating line with points is the genetic load $L$, the second line with the points is the frequency of allele $B$ and the second plain line, confounded with the abscissa at zero is the heterozygote deficiency index $F_{\text {IS }}$. (A) "focused choice for good genes" strategy with parameter values: $q(0)=10^{-6}, h=0.3, s=0.1, \mu=0.01$ and $c=0$. (B) "broad choice for good genes" strategy with $q(0)=10^{-4}, h=0.3, s=0.1, \mu=0.01$ and $c=0$. (C) "focused choice for good genes" strategy of for $q(0)=0.5, h=0.3, s=0.1, \mu=0.01$ and $c=0.005$. (D) "broad choice for good genes" strategy with same parameter values as in $(\mathrm{C})$.

females even in the zone of overdominance, where heterozygotes have a fitness advantage over either type of homozygote. This result was first noted by Irwin and Taylor (2000). Females do not benefit from favouring heterozygous males as mates, whether the attractiveness of heterozygotes is in some way directly determined by females, or mediated via improved condition of heterozygotes that in turn results in enhanced sexual displays. Unlike in the good genes scenarios above, a preference for heterozygosity cannot invade even if female choice is costfree.

Why do we and others (Partridge, 1983; Irwin and Taylor, 2000) obtain such a strong negative result, yet we know that females mating with heterozygote males produce more heterozygote offspring than if they where mating randomly (Mitton et al., 1993)? First, it is instructive to see how Mitton et al. (1993)'s result arises. Consider a population of $A A, A a$ and $a a$ females that mate randomly. Half of the offspring of $A a$ will always be heterozygotes (Eq. (11)). However, $A A$ and $a a$ females, if they mate randomly, will not achieve this high proportion of heterozygous offspring, unless the frequency of allele $A$ is exactly $\frac{1}{2}$ (Eq. (14)). Thus, heterozygous males are more likely to bear heterozygous offspring than are homozygous males. However, this correlation does not imply that a prospective female gains by favouring heterozygous individuals as mates, even under overdominance. First, consider symmetric overdominance in the absence of mutations, which predicts that the equilibrium frequency of allele $A$ under random mating is $\hat{p}=\frac{1}{2}$. With this frequency, random mating and a preference for heterozygotes - or, in fact, any mating preference that does not take into account the female's own genotype - both result in exactly the same proportions of genotypes in offspring of all types of matings. Assuming no choice costs, the choice allele and the random-mating allele are therefore selectively neutral (Irwin and Taylor, 2000).

By contrast, when the overdominance is not symmetric, selection under random mating produces asymmetrical genotype frequencies: $A A$ individuals have superior fitness relative to $a a$, which generates a deficiency in the number of $a$ alleles and $a a$ genotypes in the population. Now the expected proportion of heterozygotes offspring by a homozygous, randomly mating mother falls below one 


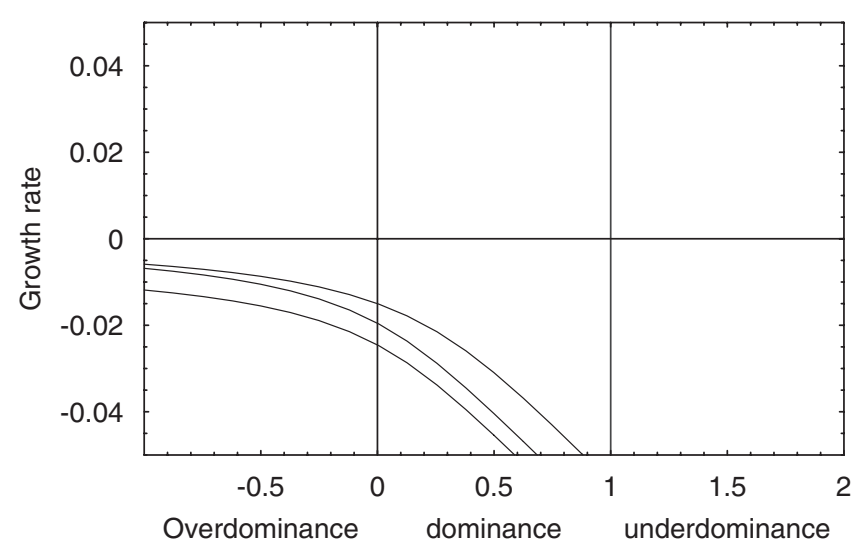

(A)
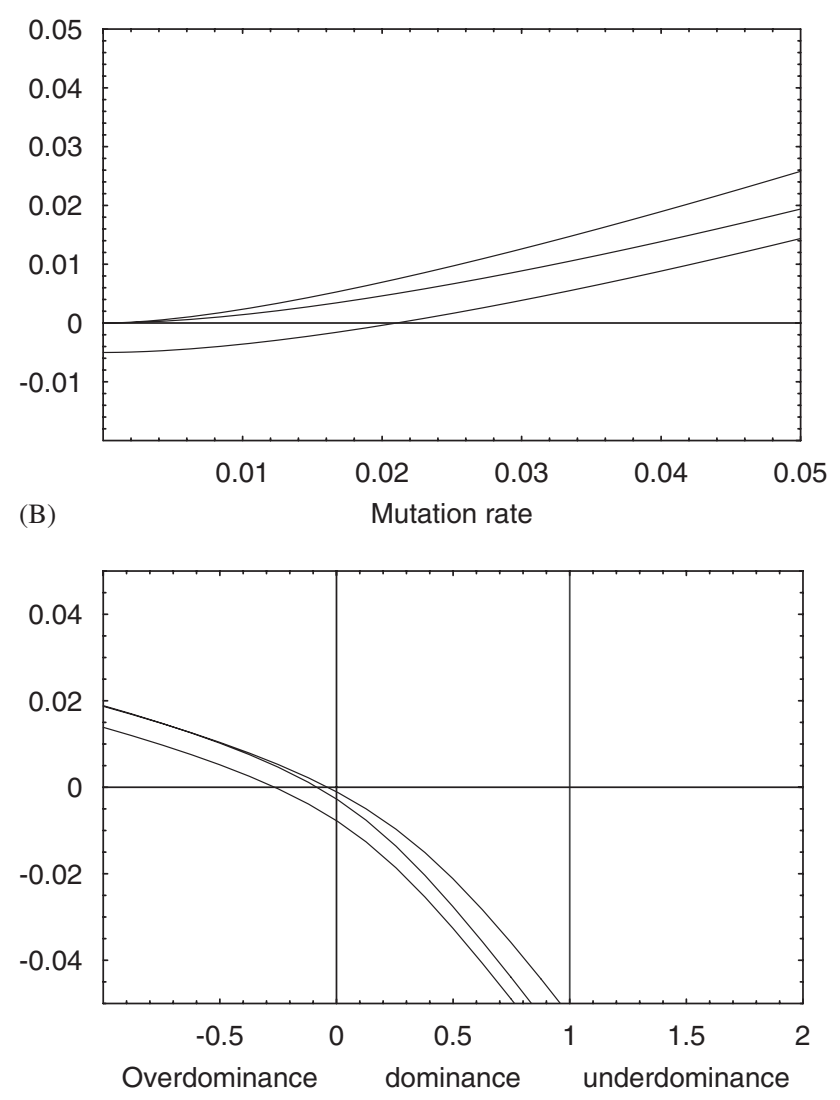

(C)

$\mathrm{h}$

Fig. 5. (A) Growth rate of the strategy choice for heterozygotes as a function of the dominance coefficient $h$. The coefficient of selection is set to $s=0.1$ and the three curves correspond, from top to bottom, to: (1) $\mu=0.01$ and $c=0$, (2) $\mu=0.005$ and $c=0$, (3) $\mu=0.005$ and $c=0.005$. A decrease in the mutation rate and an increase in the cost of choice decreases the growth rate. (B) Growth rate of the strategy choice for heterozygotes under symmetric overdominance as a function of the mutation rate. The three curves correspond, from top to bottom, to: (1) $s=0.1$ and $c=0$, (2) $s=0.05$ and $c=0$, (3) $s=0.1$ and $c=0.005$. (C) Growth rate given for the strategy disassortative mating as a function of the dominance coefficient $h$. The parameters are the same as for (A).

half, while heterozygous mothers still produce $\frac{1}{2}$ heterozygous offspring regardless of their mating strategy. Can a choosy female, who under these conditions preferentially mates with heterozygote males, improve the fitness of her progeny? As shown by Irwin and Taylor (2000), the answer is no: despite overdominance, the choice allele cannot invade the population because the increased production of $A a$ offspring also automatically associates with increased production of $a a$, at the expense of $A A$ offspring that were assumed more fit than $a a$. Thus, the redistribution of genotypes creates more of the less fit homozygote $a a$ than are created under random mating. (Randomly mating females would end up more often producing $A A$ than $a a$ females, due to the greater frequency of the $A$ allele.) This outweighs the benefit of producing more heterozygotes, unless overdominance is symmetric - but then we are back at the expectation that the frequency of $A$ is $\frac{1}{2}$, which in turn means that any mate choice strategy produces the same offspring distribution and is thus selectively neutral.

Although the above argument (and Fig. 5A) draws a bleak picture regarding the evolution of preferences for heterozygous males, there is a way out: if the frequency of $A$ can differ from $\frac{1}{2}$ while symmetric overdominance is retained, the preference for heterozygous males can be selected for. Biased mutation rates can produce such a case. To summarize: if symmetric overdominance combines with a biased mutation rate, resulting in an initial frequency of allele $A$ different of one half (Eq. (9)), the benefit of creating more heterozygote genotypes is not destroyed by overproduction of less fit homozygotes, because both homozygotes have the same fitness. This can lead to an increase of the average viability of offspring. This intuition is confirmed in Fig. 5B where we investigated the growth rate of the choice of heterozygote gene under different mutation rates determining unequal initial frequencies of the alleles at the condition-determining locus, and a numerical exploration predicts that such a preference can increase to fixation (Fig. 6A). Thus, a preference for heterozygous mates is possible to achieve. This is a situation that does not involve any "good gene" because both alleles have the same fitness, but it appears to require rather special conditions to evolve.

Disassortative mating evolves more easily (Fig. 5C). The gene determining disassortative mating is able to invade the population in the overdominance zone. This is unsurprising, since the change in the distribution of genotypes creates additional heterozygotes without suffering from the problem experienced by the blind preference for heterozygotes, i.e. overproduction of the wrong kind of homozygotes. This strategy thus results in a direct increase in mean offspring viability, as long as there is overdominance, and creates conditions under which females can benefit from favouring 'complementary' genotypes. The analysis of the dynamical model confirms qualitatively the insights gained by the invasion model. Interestingly, however, the spread of a preference for disassortative mating quickly slows down after an initial invasion period and the frequency of the choice allele never exceeds that of the random mating allele (Fig. 6B-D). This results in a stable polymorphism between the disassortative mating allele and the random mating allele. This polymorphism is a 

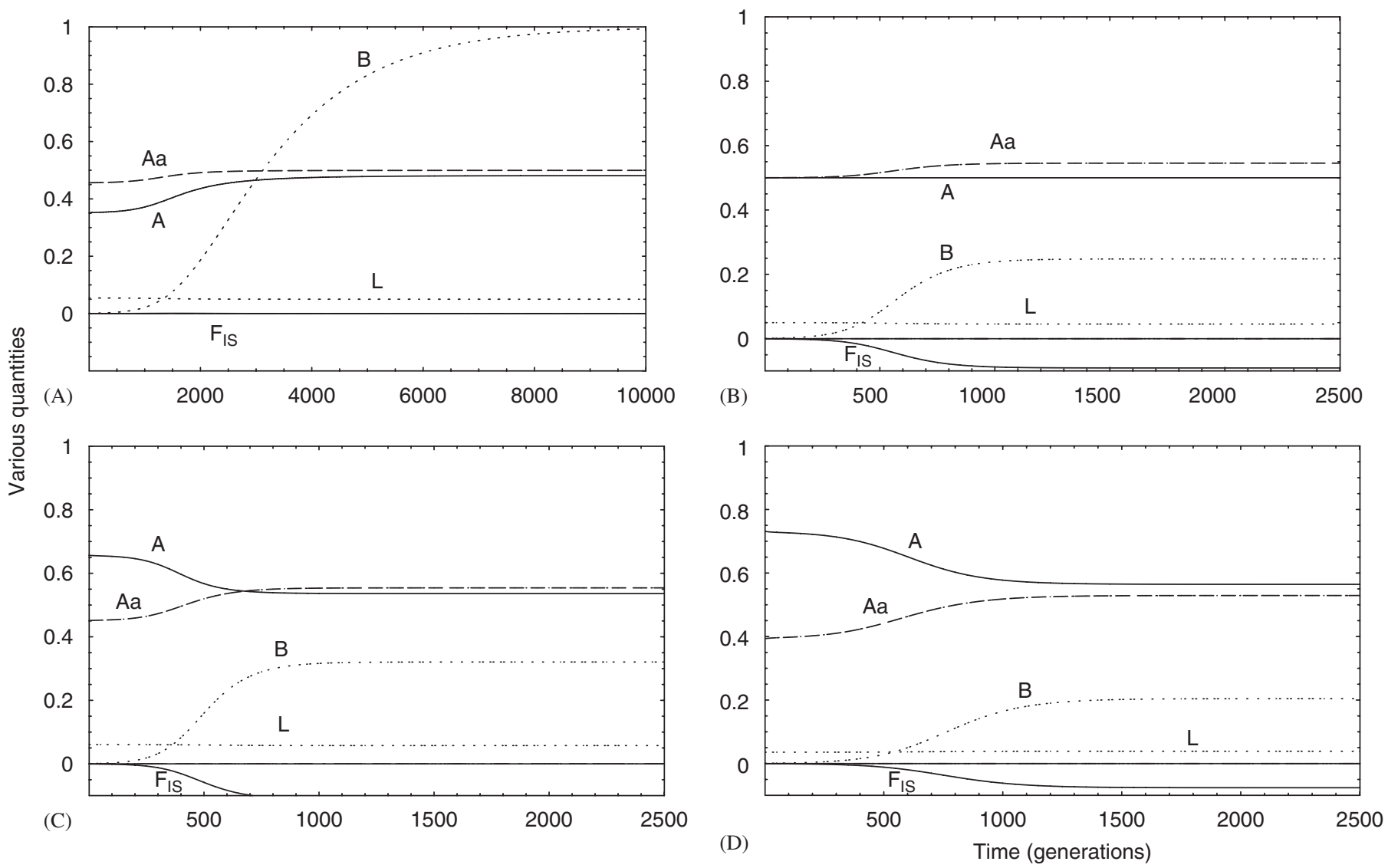

Fig. 6. Dynamic of various quantities as a function of time since the introduction of the choice allele $B$ at frequency $q(0)$ in a population at equilibrium frequency $\hat{p}$ of allele $A$ at the viability locus under random mating (Eq. (7)). The plain line correspond to the frequency of allele $A$ at the viability locus, the dashed line is the frequency of heterozygotes $A a$, the line with points is the genetic load $L$, the second line with points is the frequency of choice allele $B$ and the second plain line is the heterozygote deficiency index $F_{\text {IS }}$. (A) Choice for heterozygotes under symmetric overdominance with parameter values $q(0)=10^{-3}, s=0.1, \mu=0$ and $c=0$. (B) Disassortative mating under symmetric overdominance with parameter values $q(0)=10^{-3}, s=0.1, \mu=0.01$ and $c=0$. (C-D) Disassortative mating under asymmetric overdominance, with parameter values $q(0)=10^{-3}, s=0.1, \mu=10^{-3}, c=0$ and $h=-1$ for $(\mathrm{C})$ while $h=-0.5$ for (D).

consequence of frequency-dependence brought about by sexual selection opposing natural selection. Producing heterozygote offspring is favoured by natural selection because they survive well, leading to selection for the compatibility choice allele. However, the mating success of heterozygote males decreases as the choice gene invades because homozygous choosy females mate with homozygotes of the other type and not with heterozygote males. This induces a negative selective pressure on the choice allele stemming from choosy females themselves. Consequently, the choice gene reaches an equilibrium determined by the balance between sexual and natural selection. Comparing Fig. 6C and D reveals that a decrease in the heterozygote advantage decreases the equilibrium frequency of the disassortative mating allele.

\subsection{Assortative mating}

Under assortative mating, females avoid producing heterozygotes. It is therefore not surprising that assortative mating, if not too costly, is favoured by selection when there is either dominance or underdominance, but it is always selected against in the overdominance case (Fig. 7A).

It is interesting to note that perfect assortative mating in our model framework is formally equivalent to selfing. Indeed, the mating matrix we obtain in this situation is strictly equivalent to the matrix for selfing as given by Nagylaki (1992, Eq. 5.7). However, selfing is known to generate inbreeding depression. So, at first glance, assortative mating might result in a cost for the choice gene since it will increase the production of homozygotes offspring in exactly the same way as do selfing. Why is then assortative mating, or equivalently selfing, so easily selected for? Actually, selfing purges the population from the deleterious allele $a$ and reduces the genetic load of the population. Therefore, the selfing/assortative mating strategy is costly for their carriers only over a few generations but subsequently beneficial through increased mean viability of offspring, a classical result of population genetic theory (Gillespie, 2004, Fig. 5.5). This long term benefit is captured by the invasion condition given by the dominant eigenvalue of the transition matrix because it gives the asymptotic growth rate of allele $B$. But this invasion condition neglects 


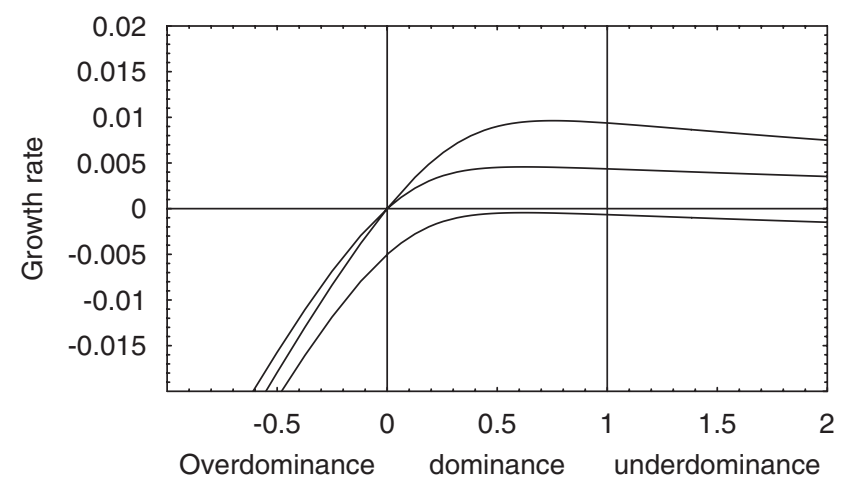

(A)

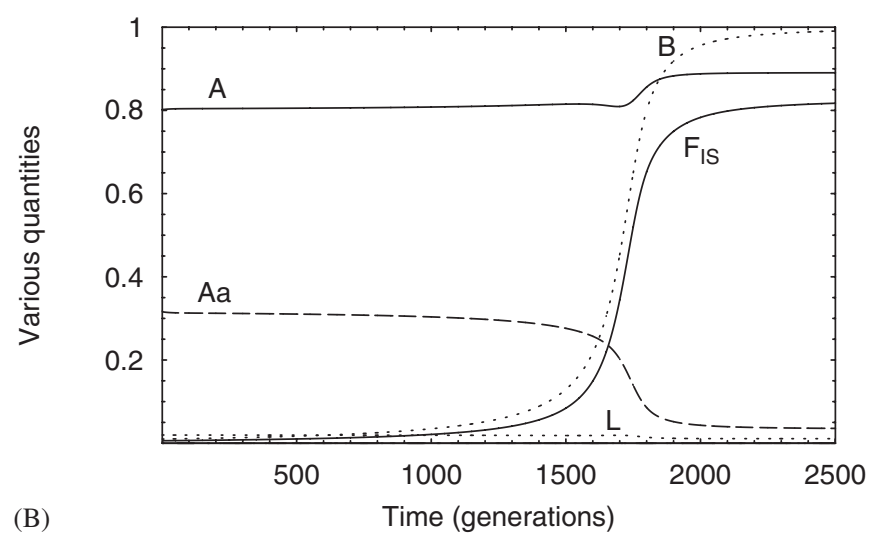

Fig. 7. (A) Growth rate for assortative mating as a function of the dominance coefficient $h$. The coefficient of selection is set to $s=0.1$ and the three curves correspond, from top to bottom, to: (1) $\mu=0.01$ and $c=0$, (2) $\mu=0.005$ and $c=0$, (3) $\mu=0.005$ and $c=0.005$. A decrease in the mutation rate and an increase in the cost of choice decreases the growth rate. (B) dynamic for assortative mating of allele frequency $A$ and $B$, of genotype $A a$ frequency, of the genetic load $L$ and of the heterozygote deficiency index. Parameter values are: $q(0)=10^{-2}, h=0.5, s=0.1, \mu=$ 0.01 and $c=0$.

the short-term fitness costs induces by selfing/assortative mating. This is the reason why in the population genetic model, there must be a threshold frequency of choice gene carriers initially set in the population to overcome the short term fitness valley resulting from the induced inbreeding depression. If the initial frequency of choice gene carriers exceeds this threshold, the choice gene spreads through fixation (Fig. 7B). While the locus subject to assortative mating follows here the same dynamics as if it were subject to selfing, it is important to recall that inbreeding affects all the loci of the genome while assortative mating only affects the set of loci under assortative mating or those that are closely linked to them. Accordingly, there is also a fundamental difference between assortative-mating and selfing.

\section{Discussion}

Genetic diversity is a central focus of all theory on mate choice based on indirect benefits: mate choice only works if there is genetic variation in male quality. At the same time, mate choice interacts with the levels of diversity that can be maintained in a population, and of particular interest are non-additive effects (Neff and Pitcher, 2005): perhaps more variation can be maintained if females favour heterozygous males rather than a fixed best genotype, or if different females have different preferences?

Our population genetic modelling allows direct comparison of several different female choice strategies and evaluates their consequences for genetic diversity at condition-dependent traits. We have analysed here the simplest situation of the world of condition-dependence, that is, a one-locus system with two viability alleles. This may appear drastic, but it allows us to derive a clear message: a given mutational input translates into very different prospects for female choice, depending on the details of genetic dominance and whether females target homozygotes or heterozygotes as mates, seek complementary alleles, or mate assortatively.

In the introduction, we alluded to a mechanism that might boost the effects of a slight mutation rate and help maintain genetic diversity at the viability locus: if female choice differs from a strict and focused choice of the best genotypes (here, $A A$ ), genetic variation is better maintained and this could maintain more female choice, i.e. females retain choosiness while tolerating bigger costs. Relaxing the strict preference for $A A$ males could be either a broad preference for good genes, where heterozygous males have comparable mating success to homozygous high quality individuals, or an actual preference for heterozygous males over any kind of homozygote. Either of these preferences turn out to spread poorly compared to a classical good genes preference, even though our results confirmed that they help to maintain genetic diversity at the viability locus. However, the potential benefit brought by this diversity is outweighed by the inaccuracy of female choice itself: compared with focused choice, broad choice for good genes produces more genetic diversity in the offspring generation, which means that female parents are less likely to produce the desired (fittest) type of offspring. The net effect is negative: even though there is more reason to choose when diversity is high, superior offspring performance when mating with superior males does not manifest itself as faithfully as in the case of focused choice. Thus, various forms of heterozygote-favouring (or variable) female choice seem to pose a problem for the theory of sexual ornamentation based on indirect benefits, rather than a solution.

There are exceptions to this conclusion, however. For example, we found that a preference for heterozygous males can evolve, but this only happens under quite specific conditions. Overdominance should be symmetric, while mutations should be biased towards one or the other allele. Our model is, of course, an oversimplification with its one condition-or viability-determining locus only, but the same logic should apply in a more general setting: mating with heterozygous males inevitably means that a fraction of offspring will be homozygous for various loci, and if some 
homozygotes are more common than others and have superior fitness, then heterozygote matings will overproduce the less fit homozygotes.

This makes it very difficult to establish a general preference for mating with heterozygote males. The recent findings that heterozygous males have superior ornaments (Marshall et al., 2003; Seddon et al., 2004; Reid et al., 2005) thus, again, pose a problem rather than a solution for the maintenance of female choice: why should they remain choosy, when dominance in ornaments means that ornaments have relatively little predictive power with respect to the condition, viability and attractiveness of offspring?

Expanding on earlier results by Partridge (1983) and Hedrick (1992), disassortative mating was found to be much more robust in our model, as long as there is overdominance. This creates conditions under which females can benefit from favouring 'complementary' genotypes. This strategy, of course, requires that the cognitive machinery (e.g. Milinski et al., 2005) is in place to allow female choice to depend on the female's own genotype. This preference invades initially with roughly similar ease as focused choice for good genes does. Interestingly, however, the spread of this strategy slows down considerably faster than a preference for good genes, even though the former maintains genetic variation while the latter depletes it. The resulting polymorphism appears to be a balance between natural and sexual selection. When choice is rare, heterozygous offspring are the fittest due to a viability benefit. But once the choice allele increases, heterozygous males have poorer mating success, which diminishes the fitness differences between offspring types, and thus improves the relative success of random mating. Thus, frequency-dependence can increase the diversity of female mating strategies (Jennions and Petrie, 1997): in addition to choosy females preferring their complementary kind, other females benefit by not being choosy at all.

Our model tracks one condition-determining locus only and it is not straightforward to predict the results if extended to multiple loci with interactions among loci and linkage disequilibria. However, the main message of our model is that one should not focus solely on the diversitymaintaining consequences of a choice rule, when predicting its evolutionary success. Any choice rule that maintains variation must do so by producing diverse kinds of offspring, and many of them will perform poorly. The latter fact has a negative impact on preference evolution. While we have not proven that the net effect will be negative if multiple loci are considered, our results certainly warn against quick conclusions based on the positive effects on diversity maintenance only.

Our model also assumes a population of infinite size and thus ignores the effects of genetic drift at either the viability or the choice locus. Given sufficiently strong directional selection, drift at the choice locus is unlikely to affect our results substantially. At the viability locus, however, genetic drift will change the starting conditions we derived (Fig. 1). In the presence of symmetric overdominance, drift should play the same role as biased mutation rate, by letting equilibrium allele frequency to differ from $\frac{1}{2}$, thus allowing preference for heterozygous males to evolve. Under dominance, genetic drift might either relax or tighten the conditions for the evolution of preference for good genes because drift can either result in an increase or a decrease in the equilibrium frequency of deleterious alleles; this equilibrium depending on the interaction between the coefficient of dominance $(h)$, the coefficient of selection $(s)$ and population size (Glémin, 2003, Fig. 3). For instance, genetic drift leading to drift load, will on average increase the frequency of the less fit allele, thus boosting the scope of selection for choice of good genes. The precise effects of drift are potentially more complicated, because drift can create linkage between the choice and the viability locus.

Finally, a cautionary note. We have assumed that male life-history depends on a pleiotropic gene that influences both the attractiveness of male offspring, and the viability of both male and female offspring. We did not model condition-dependence explicitly by seeking optimal life history reaction norms of a male to his own condition (Nur and Hasson, 1984; Getty, 1998; Kokko, 1998), nor did we derive gene frequency changes in different environments despite the importance of $G \times E$ interactions for the operation of sexual selection (e.g. David et al., 2000; Jia et al., 2000; Proulx, 2001; Welch, 2003; Hunt et al., 2004). Nevertheless, we suspect that condition-dependent sexual signalling and its interaction with genetic and environmental variation in condition makes researchers face the same dilemma that became evident in our simplified version: the very mechanisms that help maintain diversity in offspring genotypes often also mean that trying to select the best genes becomes a very inaccurate business. Condition-dependence typically means that there is a large environmental component to a male's appearance (Griffith et al., 1999; Kotiaho et al., 2001). Studies reporting such effects have even promoted this effect to the status of a (partial) resolution of the lek paradox (Kotiaho et al., 2001). Our results lead us to echo Greenfield and Rodriguez (2004) worry that there is another, somewhat neglected side to the coin: a large environmental component to a male's condition is simply another way to make females less certain that their preference leads to the desired genotypes in offspring (see also Danielson-François et al., 2006). Future studies, both theoretical and empirical, should investigate how choosiness can be maintained when dominance in sexually selected traits means that these traits have relatively little predictive power with respect to the fitness and attractiveness of offspring.

\section{Acknowledgments}

We wish to thank Luc Bussière, Michael Jennions, Jan Lindström and Jane Reid for discussions and comments, and the Academy of Finland for funding. 


\section{Appendix A}

\section{A.1. Mutation matrix}

The mutation matrix with elements $u(l \mid i)$ giving the probability that an offspring inheriting genotype $i$ will actually be of genotype $l$ after mutation reads

$\mathbf{U}=\left(\begin{array}{ccc}(1-\mu)^{2} & (1-\mu) v & v^{2} \\ 2(1-\mu) \mu & (1-\mu)(1-v)+\mu v & 2(1-v) v \\ \mu^{2} & \mu(1-v) & (1-v)^{2}\end{array}\right)$.

\section{A.2. Dynamic of the choice gene: population genetic model}

Here, we describe the elements of the transition matrix $\mathbf{T}$ of the genotype frequencies $\mathbf{p} \equiv\left(p_{A A B}, p_{A a B}, p_{a a B}, p_{A A b}\right.$, $\left.p_{A a b}, p_{a a b}\right)$ given in the main text. Notice first that the fertility of a choosy female is $1-c$, that of a random mating female is 1 and that the mean fertility is designated by $\bar{f}$. Then, the transition matrix of genotype frequencies can then be written

$\mathbf{T}=\frac{1}{\bar{f}}\left(\begin{array}{cc}(1-c) \mathbf{U} \mathbf{M}_{\mathbf{B}} \mathbf{W} & \mathbf{U R}_{\mathbf{B}} \mathbf{W} \\ (1-c) \mathbf{U} \mathbf{M}_{\mathbf{b}} \mathbf{W} & \mathbf{U R}_{\mathbf{b}} \mathbf{W}\end{array}\right)$,

where the subscript $\mathbf{B}$ and $\mathbf{b}$ of the mating matrices emphasize the allele at the choice gene locus carried by the offspring produced by the matings described by the elements of the respective matrices. Accordingly, the elements of matrix $\mathbf{M}_{\mathbf{B}}$ stand for the production of juvenile choice gene carriers of the different genotypes at the condition-determining locus by female choice gene carriers. These elements are

$\operatorname{Pr}(i B \mid j B)=\sum_{k} \operatorname{Pr}(i B \mid k, j B) \operatorname{Pr}(k \mid j B)$,

where the sum runs over all possible male genotypes while $i$ and $j$ designate the genotype at the condition-determining locus. The first term in this sum is the probability that a female with genotype $j B$ which has mated with a male with genotype $k$ produces an offspring of genotype $i B$. The choice gene of an offspring is randomly sampled from one of the parental choice gene, for instance $\operatorname{Pr}(A A B \mid A A b$, $A A B)=\frac{1}{2}$. The second term in the sum is the probability that a female with genotype $j B$ mates with a male with genotype $k$ and is given by

$\operatorname{Pr}(k \mid j B)=\frac{p_{k}^{\mathrm{s}} \psi(k \mid j B)}{\sum_{r} p_{r}^{\mathrm{s}} \psi(r \mid j B)}$,

where $\psi(k \mid j B)$ is the probability that a female with genotype $j B$ accepts a male with genotype $k$ for mating. The elements of the matrix $\mathbf{M}_{\mathbf{b}}$ are

$\operatorname{Pr}(i b \mid j B)=\sum_{k} \operatorname{Pr}(i b \mid k, j B) \operatorname{Pr}(k \mid j B)$.
The random mating matrices $\mathbf{R}_{\mathbf{B}}$ and $\mathbf{R}_{\mathbf{b}}$ are obtained similarly but by letting $\psi(k \mid j B)=1$ and $\psi(k \mid j b)=1$ for all male and female genotypes.

\section{References}

Ahtiainen, J.J., Alatalo, R.V., Mappes, J., Vertainen, L., 2004. Decreased sexual signalling reveals reduced viability in small populations of the drumming wolf spider Hygrolycosa rubrofasciata. Proc. R. Soc. London B 271 (1550), 1839-1845.

Aspi, J., 2000. Inbreeding and outbreeding depression in male courtship song characters in Drosophila montana. Proc. R. Soc. London B 271 (1550), 1839-1845.

Brown, J.L., 1997. A theory of mate choice based on heterozygosity. Behav. Ecol. 8 (1), 60-65.

Bürger, R., 2000. The Mathematical Theory of Selection, Recombination and Mutation. Wiley, New York.

Caswell, H., 2001. Matrix Population Model. Sinauer, MA.

Charlesworth, D., Morgan, M., Charlesworth, B., 1990. Inbreeding depression, genetic load, and the evolution of outcrossing rates in a multilocus system with no linkage. Evolution 44, 1469-1489.

Colegrave, N., Kotiaho, J.S., Tomkins, J.L., 2002. Mate choice or polyandry: reconciling genetic disassortative mating and good genes sexual selection. Evol. Ecol. Res. 4 (6), 911-917.

Danielson-François, A., Kelly, J., Greenfield, M., 2006. Genotype x environment interaction for male attractiveness in an acoustic moth: evidence for plasticity and canalization. J. Evol. Biol. 19, 532-542.

David, P., Bjorksten, T., Fowler, K., Pomiankowski, A., 2000. Conditiondependent signalling of genetic variation in stalk-eyes flies. Nature 406 (6792), 186-188.

Eshel, I., Sansone, E., Jacobs, F., 2002. A long-term genetic model for the evolution of sexual preference: the theories of Fisher and Zahavi reexamined. J. Math. Biol. 45 (1), 1-21.

Gavrilets, S., 2004. Fitness Landscapes and the Origin of Species. Princeton University Press, Princeton.

Getty, T., 1998. Handicap signalling: when fecundity and viability do not add up. Anim. Behav. 56, 127-130.

Gillespie, J., 2004. Population Genetics: A Concise Guide. Johns Hopkins, Baltimore \& London.

Glémin, S., 2003. Are deleterious mutations purged? Drift versus nonrandom mating. Evolution 57 (12), 2678-2687.

Greenfield, M.D., Rodriguez, R.L., 2004. Genotype-environment interaction and the reliability of mating signals. Anim. Behav. 68, 1461-1468.

Griffith, S.C., Owens, I.P.F., Burke, T., 1999. Environmental determination of a sexually selected trait. Nature 400 (6742), 358-360.

Hartl, D., Clark, A.G., 1997. Principles of Population Genetics. Sinauer, MA.

Hedrick, P.W., 1992. Female choice and variation in the major histodisassortative mating complex. Genetics 132 (2), 575-581.

Höglund, J., Piertney, S.B., Alatalo, R.V., Lindell, J., Lundberg, A., Rintamäki, P.T., 2002. Inbreeding depression and male fitness in black grouse. Proc. R. Soc. London B 269 (1492), 711-715.

Hunt, J., Bussière, L.F., Jennions, M.D., Brooks, R., 2004. What is genetic quality? Trends Ecol. Evol. 19 (6), 329-333.

Irwin, A., Taylor, P., 2000. Heterozygote advantage and the evolution of female choice. Evol. Ecol. Res. 2, 119-128.

Jennions, M.D., Petrie, M., 1997. Variation in mate choice and mating preferences: a review of causes and consequences. Biol. Rev. Cambridge Philos. Soc. 72 (2), 283-327.

Jennions, M.D., Petrie, M., 2000. Why do females mate multiply? A review of the genetic benefits. Biol. Rev. 75 (1), 21-64.

Jia, F.Y., Greenfield, M.D., Collins, R.D., 2000. Genetic variance of sexually selected traits in waxmoths: maintenance by genotype $\mathrm{x}$ environment interaction. Evolution 54 (3), 953-967.

Kirkpatrick, M., Ryan, M.J., 1991. The evolution of mating preferences and the paradox of the lek. Nature 350 (6313), 33-38 (fA693 Nature). 
Kokko, H., 1998. Good genes, old age and life history trade-offs. Evol. Ecol. 12 (6), 739-750.

Kokko, H., Brooks, R., McNamara, J.M., Houston, A.I., 2002. The sexual selection continuum. Proc. R. Soc. London B 269 (1498), 1331-1340.

Kokko, H., Jennions, M.D., Brooks, R., 2006. Unifying and testing models of sexual selection. Ann. Rev. Ecol., Evol. Syst. 37, 43-66.

Kotiaho, J.S., Simmons, L.W., Tomkins, J.L., 2001. Towards a resolution of the lek paradox. Nature 410 (6829), 684-686.

Lynch, M., Walsh, B., 1998. Genetics and Analysis of Quantitative Traits. Sinauer, MA

Marshall, R.C., Buchanan, K.L., Catchpole, C.K., 2003. Sexual selection and individual genetic diversity in a songbird. Proc. R. Soc. London B 270, S248-S250.

Maynard Smith, J., 1956. Fertility, mating behaviour and sexual selection in Drosophila subobscura. J. Genet. 54, 261-279.

Mays, H.L., Hill, G.E., 2004. Choosing mates: good genes versus genes that are a good fit. Trends Ecol. Evol. 19 (10), 554-559.

Milinski, M., Griffiths, S., Wegner, K.M., Reusch, T.B.H., HaasAssenbaum, A., Boehm, T., 2005. Mate choice decisions of stickleback females predictably modified by MHC peptide ligands. Proc. Natl Acad. Sci. USA 102 (12), 4414-4418.

Mitton, J.B., Schuster, W.S.F., Cothran, E.G., de Fries, J.C., 1993. Correlation between the individual heterozygosity of parents and their offspring. Heredity 71, 59-63.

Møller, A.P., Alatalo, R.V., 1999. Good-genes effects in sexual selection. Proc. R. Soc. London B 266 (1414), 85-91.

Nagylaki, T., 1992. Introduction to Theoretical Population Genetics. Springer, New York

Neff, B.D., Pitcher, T.E., 2005. Genetic quality and sexual selection: an integrated framework for good genes and compatible genes. Mol. Ecol. 14 (1), 19-38.

Nur, N., Hasson, O., 1984. Phenotypic plasticity and the handicap principle. J. Theor. Biol. 110 (2), 275-297.

Partridge, L., 1983. In: Bateson, P. (Ed.), Mate Choice. Cambridge University Press, Cambridge, pp. 227-255.

Proulx, S.R., 2001. Female choice via indicator traits easily evolves in the face of recombination and migration. Evolution 55 (12), 2401-2411.
Qvarnström, A., Pärt, T., Sheldon, B.C., 2000. Adaptive plasticity in mate preference linked to differences in reproductive effort. Nature 405 (6784), 344-347.

Qvarnström, A., Brommer, J., Gustafsson, L., 2006. Testing the genetics underlying the co-evolution of mate choice and ornament in the wild. Nature 441 (7089), 84-86.

Reid, J.M., Arcese, P., Keller, L.F., 2003. Inbreeding depresses immune response in song sparrows (Melospiza melodia): direct and intergenerational effects. Proc. R. Soc. London B 270 (1529), 2151-2157.

Reid, J.M., Arcese, P., Cassidy, A.L.E.V., Marr, A.B., Smith, J.N.M., Keller, L.F., 2005. Hamilton and Zuk meet heterozygosity? Song repertoire size signals inbreeding and immunity in song sparrows (Melospiza melodia). Proc. R. Soc. London B 272, 481-488.

Reinhold, K., 2002. Modelling the evolution of female choice strategies under inbreeding conditions. Genetica 116 (2-3), 189-195.

Rowe, L., Houle, D., 1996. The lek paradox and the capture of genetic variance by condition dependent traits. Proc. R. Soc. London B 263 (1375), 1415-1421.

Saccheri, I.J., Lloyd, H.D., Helyar, S.J., Brakefield, P.M., 2005. Inbreeding uncovers fundamental differences in the genetic load affecting male and female fertility in a butterfly. Proc. R. Soc. London B 272 (1558), 39-46.

Seddon, N., Amos, W., Mulder, R.A., Tobias, J.A., 2004. Male heterozygosity predicts territory size, song structure and reproductive success in a cooperatively breeding bird. Proc. R. Soc. London B 271 (1550), 1823-1829.

Tomkins, J.L., Radwan, J., Kotiaho, J.S., Tregenza, T., 2004. Genic capture and resolving the lek paradox. Trends Ecol. Evol. 19 (6), 323-328.

Tregenza, T., Wedell, N., 2000. Genetic disassortative mating, mate choice and patterns of parentage: invited review. Mol. Ecol. 9 (8), 1013-1027.

Welch, A.M., 2003. Genetic benefits of a female mating preference in gray tree frogs are context-dependent. Evolution 57 (4), 883-893.

Zeh, J.A., Zeh, D.W., 2003. Toward a new sexual selection paradigm: polyandry, conflict and indisassortative mating (Invited article). Ethology 109 (12), 929-950. 